\title{
Dilemma of clinician; making clinical decisions sans supportive laboratory findings
}

\author{
Wickramasinghe DSA, Weerathunga DN, Nilanga WFC, Lekamwasam JDVC \\ Teaching Hospital, Karapitiya, Galle, Sri Lanka.
}

Correspondence: Dr. Sugeesha Wickramasinghe

e-mail: sugeesha@gmail.com

\section{Introduction}

Thyrotoxicosis is a prevalent condition with diverse clinical presentations. It is typically associated with low TSH and high free thyroxin levels. Making the diagnosis of thyrotoxicosis in the absence of typical TSH and thyroxin changes is a daunting task and demands very accurate interpretation of clinical information. Here we describe an atypical presentation of thyrotoxicosis where delayed diagnosis led to a potentially fatal outcome.

\section{Case report}

A 22 year-old mother of a child with a meningomyelocele, presented with fever, difficulty in breathing and productive cough of 4 days. She also complained of noticeable weight loss despite normal appetite over the preceding 5 years. Her bowel habits were normal and there was no indication of steatorrhoea during this illness. She did not complain of chronic cough or evening pyrexia and she denied experiencing tremors, palpitation or excessive sweating.

Initial examination showed an emaciated woman (BMI - $16.4 \mathrm{~kg} / \mathrm{m}^{2}$ ) who was febrile, tachypnoeic with a respiratory rate of $32 / \mathrm{min}$ and pulse rate of $120 /$ min. Her breath sounds were vesicular. Bilateral coarse crepitations were audible in middle and lower zones, bilaterally. Her neck muscles were weak and she had to support the chin to lift the head. Neurological examination revealed generalised muscle wasting, weakness with Grade 4 power and exaggerated tendon reflexes.

Her initial investigations revealed White cell count of $22.15 \times 10^{9} / \mathrm{L}$ (neutrophils - 85\%), $\mathrm{Hb}-10.7 \mathrm{mg} / \mathrm{dL}$,
Platelet - 279x10 $/ \mathrm{L}, \mathrm{CRP}-48 \mathrm{mg} / \mathrm{dL}, \mathrm{ESR}-12 \mathrm{~mm}$, $\mathrm{Na}^{+}-140 \mathrm{mmol} / \mathrm{L}, \mathrm{K}^{+}-3.9 \mathrm{mmol} / \mathrm{L}$. Liver function tests on admission revealed AST - 81U/L, ALT $76 \mathrm{U} / \mathrm{L}$, Serum protein - 66g/L, Serum albumin $41 \mathrm{~g} / \mathrm{L}$, Total bilirubin - 6.1 umol/L. Thyroid Function Tests (TFT) were normal in 3 occasions (done from two different labs) with a TSH - $1.75 \mathrm{mIU} / \mathrm{L}(0.47$ / 4.7), free $\mathrm{T}_{4}-14.9 \mathrm{pmol} / \mathrm{L}(10-28.2)$. Her stool analysis revealed the presence of fat globules but her stool culture was normal.

While waiting for the lab results. Patient was thought to have community-acquired pneumonia and she was treated with oxygen inhalation, intravenous cefotaxime and clarithromycin. She, however, developed type II respiratory failure requiring intubation and ventilation. Although her chest infection resolved completely she could not be weaned off ventilator due to poor respiratory muscle effort. Investigations at this stage showed CPK level of 204U/L (24 - 195) and EMG was suggestive of myopathy. Her serum vitamin D3 was low $24.7 \mathrm{nmol} / \mathrm{L}$ (75 - 150). She had a fasting blood sugar value of $68 \mathrm{mg} / \mathrm{dL}$ with negative retro-viral antibodies.

Although thyrotoxicosis was the most plausible etiology of her clinical picture, repeatedly normal TSH and thyroxin levels prevented us treating her with antithyroid drugs which are known to have serious adverse effects. Gradual deterioration of her condition, however, forced us to accept our clinical instinct despite serious concerns among the other members of the multidisciplinary team about our clinical judgment. Accordingly, she was started on oral propylthiouracil $350 \mathrm{mg} /$ day, verapamil $40 \mathrm{mg}$ tid. She was treated with intravenous vitamin D $10000 \mathrm{IU}$ single dose prior to antithyroid therapy 
because of low Vitamin D level but did not lead to any clinical improvement. She gradually improved allowing us to extubate her and subsequently discharged on propylthiouracil, verapamil, $1 \alpha$ cholecalciferol and calcium lactate.

\section{Discussion}

Thyrotoxicosis is generally associated with elevated free $\mathrm{T}_{4} / \mathrm{T}_{3}$ with low TSH levels. Our patient had normal TFT in three instances. Despite of repeatedly normal TFT, presence of symptoms and signs such as persistent tachycardia, weight loss, myopathy, exaggerated reflexes, fat globules in SFR, led to the provisional diagnosis of thyrotoxicosis.

Many case reports have been published revealing the presence of thyrotoxicosis with normal TFT $(1,2)$. There have been cases with resistant thyrotoxicosis with normal TFT where careful clinical evaluation has led to the correct diagnosis and management of the patient. These patients have been monitored with clinical parameters (weight gain, pulse rate, improvement and resolution of other thyrotoxic symptoms). Drug doses have been adjusted according to the clinical rather than biochemical response and patients have completely recovered.

It has been shown that $46 \%$ of the patients with thyrotoxicosis have isolated fat globules in their faeces due to an unknown reason (3). Furthermore, thyrotoxic patients have low vitamin D level again due to unknown reasons (4) and calcium and vitamin D supplements have led to a better response to antithyroid drugs (5). Observational studies have shown that there is an increase incidence of meningomyelocoele among children born to thyrotoxic mothers. It has been found out that multiple genetic mutations are responsible for this association (6).
In addition to clinical parameters, patient had biochemical parameters supportive of thyrotoxicosis such as low vitamin D levels, fat globules in stool which are widely reported in the literature. She also had a child with a meningomyelocoele which is a well explained association.

\section{Conclusions}

It is possible for patients with clinically overt thyrotoxicosis to have normal TSH and thyroxin levels. In this situation, subtle clinical features can help clinical decision making and rescue the patient's life.

\section{References}

1. John K Amory, Irl B Hirsch. Hyperthyroidism from autoimmune thyroiditis in a man with type 1 diabetes mellitus: a case report. Journal of Medical Case Reports 2011; 5: 277.

2. Taimur Saleem, Aisha Sheikh, Qamar Masood. Resistant Thyrotoxicosis in a Patient with Graves Disease: A Case Report, Journal of Thyroid Research 2011. (Article ID 649084)

3. Dinesh K. Dhanwal. Vitamin D deficiency in hyperthyroidism. Indian Journal of Medical Research 2012; 136: 311.

4. Vitamin D deficiency and its associations with thyroid disease, Mackawy AMH, Al- ayed BM, Al-rasheedi BM, International Journal of Health Sciences 2013; 7: 267-75.

5. Veletzas C. Vitamin D metabolism in thyrotoxicosis. Therapeutic aspects derived from an old observation. International Journal of Clinical Practice 2009; 63(8): 1265.

6. Relations between thyrotoxicosis and meningomyelocoele, http://biograph.be/concept/graph/C0040156/C0025312 